\title{
Widening of Laths in Bainite
}

\begin{abstract}
JIAQING YIN, MATS HILLERT, and ANNIKA BORGENSTAM
Units of bainite in Fe-C alloys from the upper temperature range inherit their shape from Widmanstätten plates of ferrite, which are lathlike. The thickness increases by long-range diffusion of carbon and the length by short-range diffusion of carbon from the advancing edge of the tip. Both have been studied extensively and are fairly well understood. Widening growth seems to have been much neglected, but a study of some aspects of widening is now presented. The present report is the last one in a series of four morphological studies of bainite, isothermally formed in $\mathrm{Fe}-\mathrm{C}$ alloys with 0.3 or 0.7 mass pct carbon, mainly in the upper temperature range. It contains a number of morphological observations made on cross sections of packets of bainite, and it elucidated a number of interesting questions about bainite and resulted in some proposals. The ferrite plates in a packet are nucleated as a group on a grain boundary, not each one separately on the side of a prior plate. Lengthening occurs by advancement of a short edge that is formed in close contact to the grain boundary. Widening of laths does not start spontaneously. It is initiated by a modification of the structure of the long edge of the lath. When it then moves, the lattice of the new ferrite is rotated relative to the ferrite formed by lengthening and the habit plane is different. In a section through the length direction, it is difficult to recognize what part of ferrite has formed by widening growth. Furthermore, it is proposed that the individual plates in a microstructure, previously used to illustrate subunits formed by repeated nucleation, were nucleated on a hidden grain boundary.
\end{abstract}

DOI: $10.1007 / \mathrm{s} 11661-017-4316-2$

(C) The Author(s) 2017. This article is an open access publication

\section{INTRODUCTION}

IT is well known that bainitic ferrite formed in the upper temperature range is lath shaped $;^{[1-3]}$ i.e., the length is much larger than the width, which, in turn, is much larger than the thickness. One may, thus, talk about broad faces, short edges, and long edges, as illustrated in Figure 1. It is evident that the growth mechanisms are different in the three directions in view of the difference in growth rates. In two recent studies $^{[4,5]}$ of bainite in $\mathrm{Fe}-\mathrm{C}$ alloys with 0.3 mass pet carbon in the upper temperature range, the emphasis was on lengthening. Bainite nucleated on grain boundaries, and long units with contact to a grain boundary were studied in order to increase the chance that the selected units were sectioned close to the length direction.

It was observed that information from sections that were not sufficiently close to the length direction could cause severe confusion if the sections were interpreted as representatives of lengthening. The situation was not as serious in the study of the upper temperature range of

JIAQING YIN, MATS HILLERT, and ANNIKA BORGENSTAM are with the Department of Materials Science and Engineering, KTH Royal Institute of Technology, Brinellvgen 23, 10044 Stockholm, Sweden. Contact e-mail: jiaqing@kth.se

Manuscript submitted May 10, 2017.

Article published online September 14, 2017 bainite, where the ferritic constituent nucleates on grain boundaries. If a particular ferritic unit is among the longest in the prepared section, one could be reasonably confident that the plane of polish is approximately parallel to the direction of lengthening. However, there is still a considerable risk that the tip of the ferrite unit in the plane of polish does not represent the growing tip. The section could have left the ferritic unit close to the tip by passing through the long edge on the side of the lath-shaped ferrite plate, and the shape of the tip in the plane of polish would be the result of widening. This is illustrated in section $\mathrm{c}$ of Figure 1. The danger of mistaking various sections as being parallel to the length direction would be more severe in the study at lower temperatures, where ferrite can nucleate intragranularly. For this reason, some attention was also paid to the shape of the long edge by studying cross sections through packets of plates.

In the study of Widmanstätten ferrite and bainitic ferrite in steels with 0.3 mass pct carbon, ${ }^{[4]}$ it was observed that cross sections of packets with ferrite plates sometimes had small, irregular protrusions on the ends of ferrite plates in the plane of polish. Of course, those ends represented the long edge of the lath-shaped plates of ferrite, as illustrated by the short sides of sections $\mathrm{d}$ through $\mathrm{f}$ in Figure 1. In other cases, there were short outgrowths on the long edges in a direction different from the initial direction of the ferrite units in the cross 


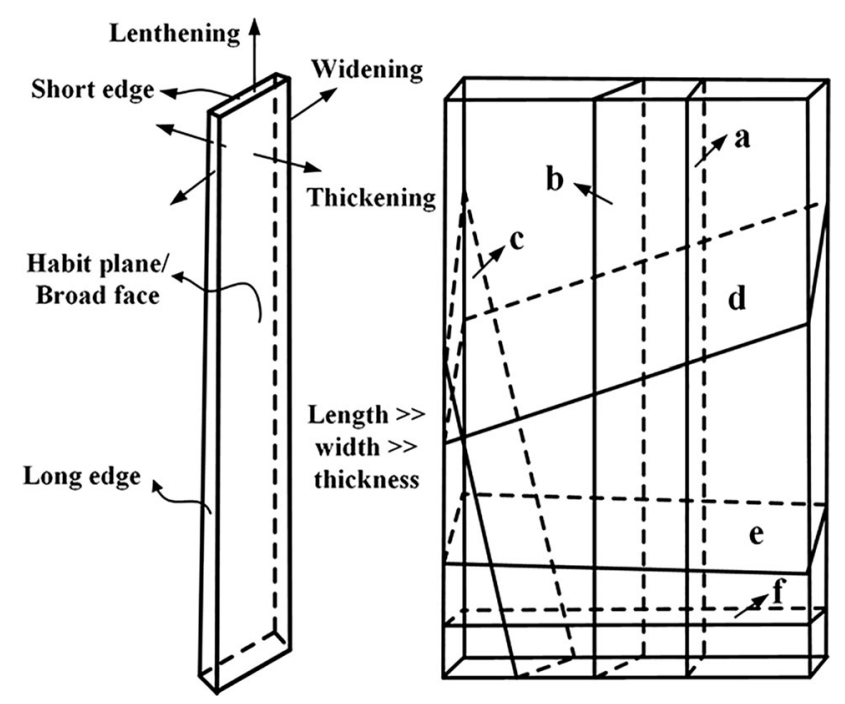

Fig. 1-Definition of growth directions of a lathlike precipitate and various sections to be observed in the plane of polish.

section. It was concluded that these outgrowths were actually sections through ridges on the long edges of the ferrite lath, which would not be recognized as abnormal in a section through the long dimension of the lath. However, in a slightly deviating direction, they gave the impression of the beginning of a side plate in a new direction. It was suggested that in an extreme case, they could be the explanation of so-called degenerate Widmanstätten plates presented by Aaronson, ${ }^{[6]}$ which had developed habit planes in two very different directions. Similar observations were made in a subsequent part of the present project on the morphology of bainite, which concerned an $\mathrm{Fe}-\mathrm{C}$ alloy with 0.7 mass pet carbon, ${ }^{[7]}$ but the report on that work focused on cases that at least looked as if they displayed the advancing tip of the unit and were, thus, interpreted as lengthwise sections. Observations that could reasonably be related to growth in the widening direction will be discussed in the present report on further work on the alloy with 0.7 mass pct carbon. For information on experimental details, reference is made to the preceding report. ${ }^{[7]}$

As a background, Figure 2 from $723 \mathrm{~K}\left(450{ }^{\circ} \mathrm{C}\right)$ is reproduced from the preceding report. ${ }^{[7]}$ It illustrates plates or packets of plates growing from one side of a grain boundary and a large number of cross sections on the other side but without contact to the same grain boundary. It was noticed that the interior of the grains was free of ferrite, as here illustrated by the lower left part of Figure 2. It was concluded that there was no intragranular nucleation of ferrite in this specimen and all the plates or packets, shown as cross sections, must then have come from a grain boundary on a different level in the specimen. That hidden grain boundary was not sectioned by the plane of polish but may have intersected the visible grain boundary not far from the plane of polish.

In a preceding report, ${ }^{[4]}$ it was shown that many ferrite particles, nucleated on the same grain boundary, often belong to a single family or to very few. In each

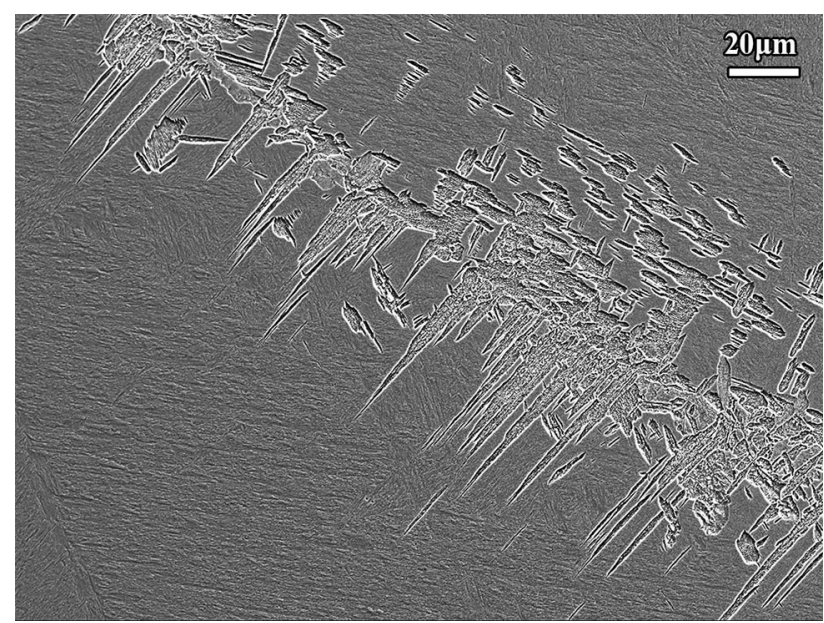

Fig. 2-Parallel packets in their long dimension on the lower side of the grain boundary and cross sections of packets on the upper side. They have started from a grain boundary above or below the plane of polish. SEM micrograph of an Fe- $0.7 \mathrm{C}$ alloy after $8 \mathrm{~s}$ at $723 \mathrm{~K}$ $\left(450^{\circ} \mathrm{C}\right)$. Reproduced from Ref. [7].

family of plates, they are parallel to each other. The plates in different cross sections in Figure 2 are parallel, and it was concluded that they all belong to a family of plates nucleated on a hidden grain boundary. The present study will mainly concern such cross sections. As long as no bainite could be seen in the interior of large austenite grains, it was concluded that there was no intragranular nucleation in that specimen.

In principle, the nucleation site and widening growth of one specific packet of units could be checked with serial sectioning and computer-aided three-dimensional (3-D) reconstruction, as has been applied for degenerate ferrite in the $\mathrm{Fe}-\mathrm{C}-\mathrm{W}^{[8]}$ and $\mathrm{Fe}-\mathrm{C}-\mathrm{Mo}^{[9]}$ systems. Similar works have also been carried out for other shapes of ferrite particles, e.g., for sawteeth, ${ }^{[10]}$ allotriomorphs, ${ }^{[11]}$ and Widmanstätten ${ }^{[10,12]}$ shapes nucleated at grain boundaries and for irregular ${ }^{[13]}$ and acicular shapes ${ }^{13,14]}$ nucleated on inclusions. All these studies concerned high-alloy Fe-C-X systems. The present work primarily aims to gain some basic understanding of widening growth of lath-shaped bainitic ferrite in the $\mathrm{Fe}-\mathrm{C}$ system with information from two-dimensional (2-D) sections and in the hope that it could stimulate 3-D works on this topic.

\section{OBSERVATIONS}

This article contains micrographs obtained with different techniques. Many of the electron micrographs contain fine details that cannot be appreciated in the printed version. The reader is encouraged to inspect the originals available in the on-line version of the article.

The electron channeling contrast imaging (ECCI) micrograph in Figure 3 from $773 \mathrm{~K}\left(500^{\circ} \mathrm{C}\right)$ shows the cross section of a packet of plates, which have almost merged by eutectoid transformation but can still be distinguished by white lines that represent either remaining austenite with high carbon content or cementite. An 


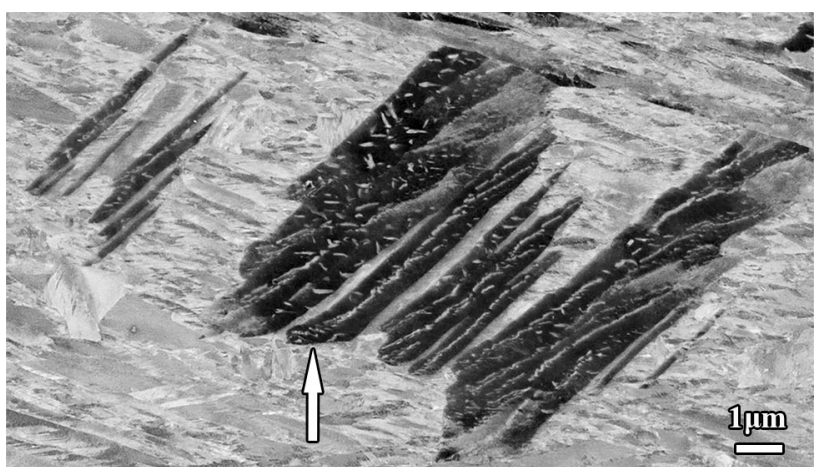

Fig. 3 - One packet sectioned close but not quite to the cross section. The arrow indicates that two ferrite plates have merged and widened in a new direction. ECCI of an Fe-0.7C alloy after $10 \mathrm{~s}$ at 773 $\mathrm{K}\left(500{ }^{\circ} \mathrm{C}\right)$.
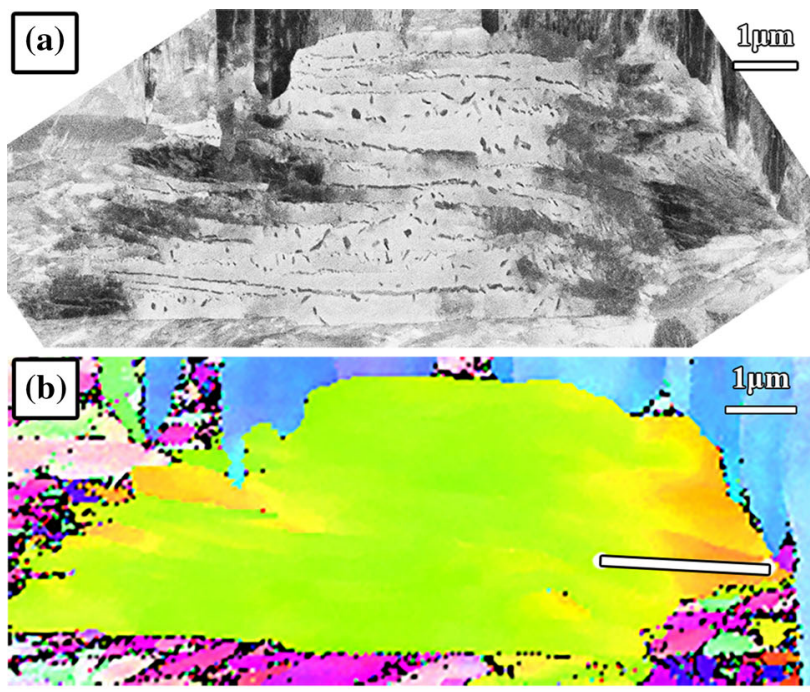

(c)

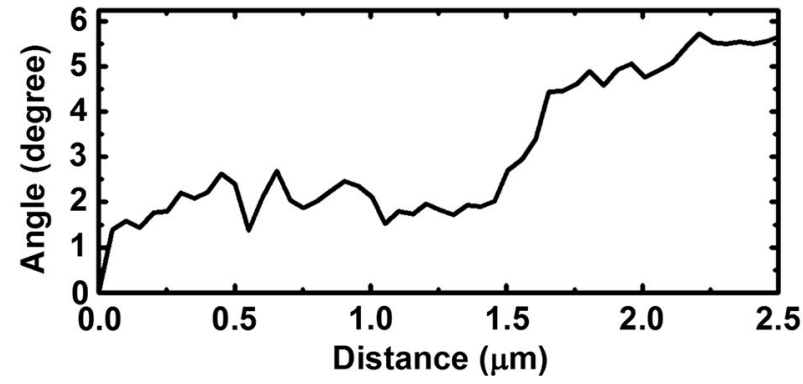

Fig. 4 - One packet of ferrite plates in an Fe-0.7C alloy after $10 \mathrm{~s}$ at $773 \mathrm{~K}\left(500{ }^{\circ} \mathrm{C}\right)$, sectioned almost perpendicular to their length dimension. (a) ECCI contrast and (b) EBSD IPF map. (c) Result of the misorientation line scan, which corresponds to (b) the white line starting from the left.

arrow marks the end of two merged plates, which seem to have increased their width by an outgrowth in a new direction. This is similar to several observations in the alloy with 0.3 mass pct carbon in the preceding study. ${ }^{[4]}$ The same phenomenon occurs on the right-hand side of a packet in Figure 4(a) from the same specimen, which is also an ECCI micrograph, but the contrasts were

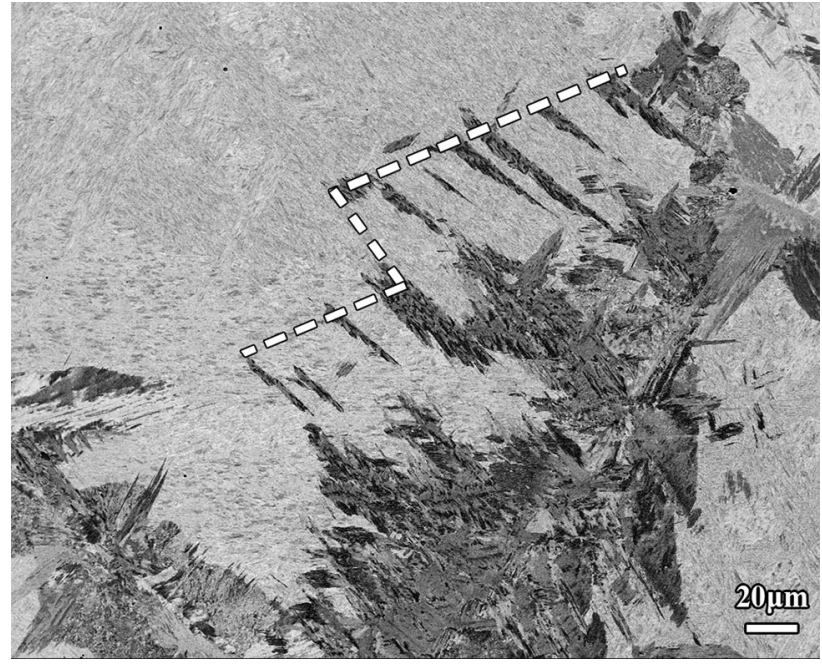

Fig. 5-Ten parallel packets of ferrite plates without contact to a grain boundary in the plane of polish. Six of them are linked by an imaginary straight line and another four by another one, possibly coherent twin boundaries. The inner part of the grain, upper left part in the figure, is free of ferrite plates, indicating that no intragranular nucleation has happened. ECCI from $773 \mathrm{~K}\left(500{ }^{\circ} \mathrm{C}\right)$ held for $10 \mathrm{~s}$ in an $\mathrm{Fe}-0.7 \mathrm{C}$ alloy.

inverted, possibly due to a difference in lattice orientation. Small black particles are cementite and ferrite is mainly white but sometimes of different shades of gray, which may only partly be caused by different lattice orientations. They may also be caused by the conditions of the polished surface. ${ }^{[15]}$ These variations seem to coincide with the bent ends of the plates. An EBSD orientation map is presented in Figure 4(b), and it confirms that the bending coincides with a rotation of the lattice. The misorientation line scan in Figure 4(c) gives about 5 deg of rotation. It is concluded that the widening process first results in a slight rotation of the lattice, which results in a different habit plane during further growth in a new direction.

When interpreting micrographs of such packets, it should be realized that they form by the lengthening growth of thin primary plates of ferrite with interspaces with remaining austenite. Such plates are seen to the left in Figure 3. Two neighboring plates of ferrite may merge by thickening, but in Figure 3, they are often separated by a white line, which may consist of cementite or sometimes of carbon-enriched austenite. It is also possible that a plate thickens on one or both sides by a cooperative eutectoid reaction, yielding a mixture of ferrite and cementite. Some plates to the left in Figure 3 have started to thicken by that process. In the middle of Figure 3, there are more well-developed examples of the eutectoid mixture. It may then be difficult to distinguish the primary plates. Units of both kinds may be regarded as bainite plates. When discussing how the transformation proceeds, it is sometimes necessary to discuss how it progresses in an austenitic interspace between two primary plates of ferrite. For the present alloy, this was discussed in a preceding report. ${ }^{[7]}$ 
The ECCI micrograph in Figure 5 from the same specimen shows 10 packets of bainite without contact to the visible grain boundary. As for the cross sections in Figure 2, it is concluded that they have nucleated on a hidden grain boundary situated on a different level in the specimen. Six of the packets seem to start from an imaginary straight line, which is indicated in the micrograph, and the other four seem to start from a parallel line. It is concluded that corresponding lines actually exist on the hidden grain boundary. Since the ferrite plates are lathlike due to a high growth rate in a particular direction and they have lengthened toward the plane of polish, the extensions of the parallel packets are still limited by a straight line but now imaginary. It is proposed that the two parallel lines on the plane of polish are projections of intersections with coherent twin boundaries in the austenite grain on the other side of the hidden grain boundary, and it seems that this intersection is particularly active to nucleate a special family of plates. The two twin boundaries may be connected by an incoherent twin boundary. The situation described here does not seem to be a unique situation, because there is a strikingly similar arrangement of Widmanstätten plates in Figure 6 from $973 \mathrm{~K}\left(700{ }^{\circ} \mathrm{C}\right)$ in an alloy with 0.3 mass pet carbon, which is reproduced from the preceding study. ${ }^{[4]}$

The ECCI micrograph in Figure 7 is a higher magnification of Figure 5, and the various shades of gray may partly reveal differences in lattice orientations but other factors may also contribute to the contrasts in the ECCI technique, as already mentioned. Better information on orientations is obtained from the EBSD orientation map in Figure 8. In the packets, there are mainly two shades of blue with an internal, dark blue band extending over their entire length. The packets of plates consist mainly of ferrite, and their boundaries are more

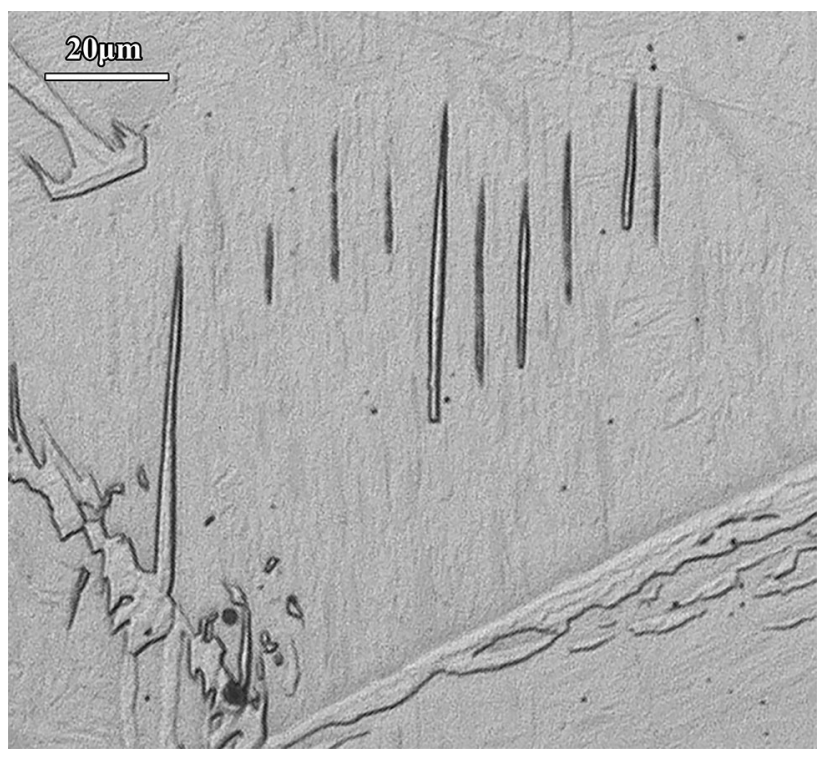

Fig. 6-Parallel Widmanstätten plates, which must have had a common origin, surprisingly similar to the parallel packets shown in Fig. 5. LOM from an Fe-0.3C-0.5Si alloy held for $10 \mathrm{~s}$ at $973 \mathrm{~K}$ $\left(700{ }^{\circ} \mathrm{C}\right)$. Reproduced from Ref. [4]. visible in the EBSD pattern quality micrograph in Figure 9, which gives the general impression of plates extending through the entire width of the packet and sloping to the right. As expected, the EBSD misorientation line scan analysis in Figures 10(a) and (b) shows that there is an orientation difference of about $6 \mathrm{deg}$ between the lattice in the dark blue part in the middle of each plate and in the light blue parts on the sides. This is similar to the case in Figure 4.

The situation is better illustrated at higher magnification in Figure 11, where the two horizontal arrows mark a band of almost horizontal, black plates that are short and appear black with the ECCI technique. They are part of a longer band marked by arrows in Figure 10(d), which falls within the dark blue band in the EBSD micrograph in Figure 10(a). It seems evident that they are parts of laths, having formed by lengthwise growth from the hidden grain boundary to the plane of polish, where they correspond to the top part of the hill in the curve of Figure 10(b). They have widened, i.e., lengthened in the plane of polish, and soon turned white in Figure 11 due to rotation of the lattice, as illustrated by the sloping sides of the hill in Figure 10(b). When reaching the lower level in Figure 10(b), they have again turned black, although the lattice orientation is different from that on top of the hill. It may further be noted that the short black parts in the middle of the plates are almost horizontal in comparison to the parts formed by widening in both directions.

Everything below the line marked by two horizontal arrows in Figure 11 was formed by widening, and detailed inspection shows that widening growth can develop in two manners. Both are affected by the fact that the interspaces between the plates turn broader as the growth direction becomes more sloping. In some areas, the interspaces transform with the cooperative mode of the eutectoid reaction. ${ }^{[2,4]}$ In other cases, a plate splits up into several thinner plates, as for the plate indicated by the vertical arrow in Figure 11, and these plates can diverge to fill a wider space and in a section may look like a broom.

Figure 12 is a higher magnification of the small packet close to the bottom of Figures 7 through 9. It may be in an earlier stage of development, because there are not many signs of rotation, which indicates that there has not yet been much widening. Two features may be noticed. In the right-hand part, there is a long horizontal plate without change of growth direction or rotation of the lattice. Actually, the same feature may be noticed in the second longest packet. Even though they are longer than the short, black plates in Figure 11, it is suggested that with their full length, they originated from the hidden grain boundary and then grew by lengthening. The other feature is common to all the packets. The dark blue band through all packets in Figure 8 bends down on the left-hand side, in particular in the second longest packet, where it actually continues to a group of short and dark blue plates that are stacked on top of each other. In addition, they are almost parallel to all the other dark blue plates. It seems that they all actually belong to the same packet. It is suggested that there was a tendency of such stacking 


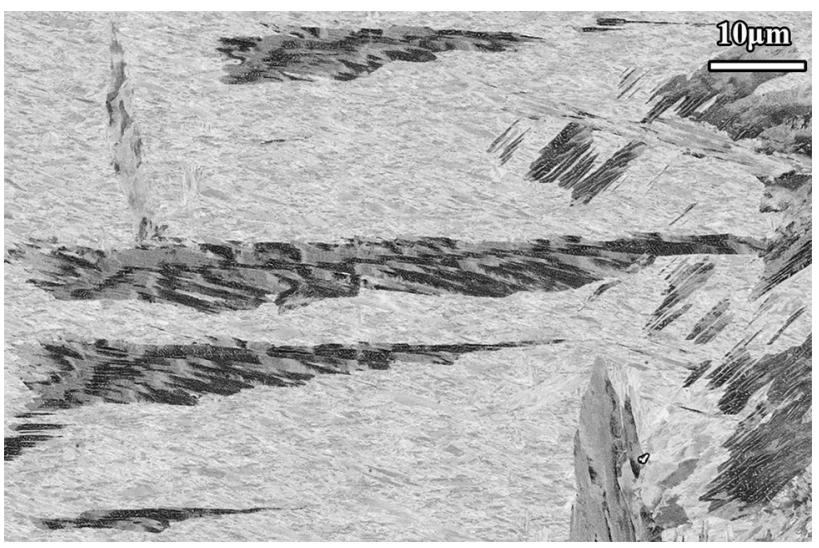

Fig. 7-Four parallel packets of ferrite plates from Fig. 5, which are now aligned horizontally. ECCI from an Fe-0.7C alloy held for $10 \mathrm{~s}$ at $773 \mathrm{~K}\left(500{ }^{\circ} \mathrm{C}\right)$.

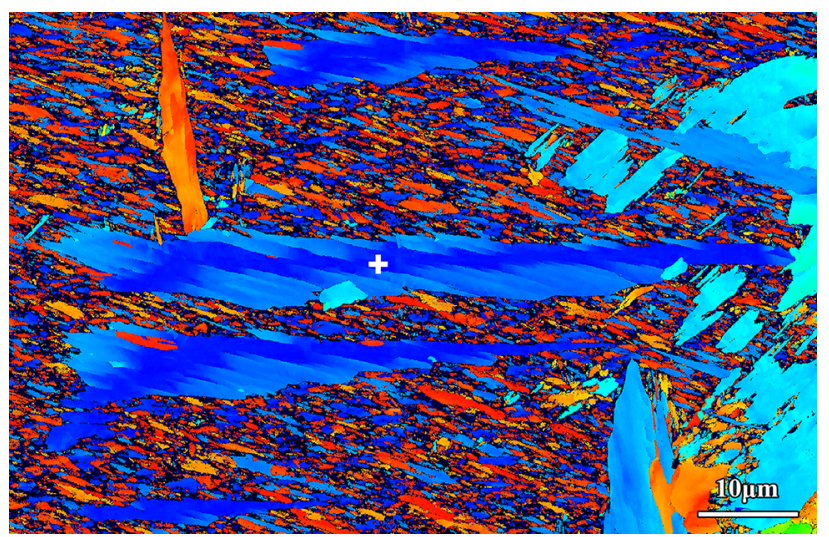

Fig. 8- Same region as Fig. 7 but with EBSD misorientation map to a reference point, marked as a white cross.

for each packet when it started to form at the twin boundary. On the other hand, the unique feature of this group of packets is that the plates are spread out and finally end with a plate that is long without showing typical signs of widening growth.

The scanning electron microscopy (SEM) micrograph in Figure 13 from $723 \mathrm{~K}\left(450^{\circ} \mathrm{C}\right)$ shows the cross section of a packet of plates, which have almost merged by cooperative eutectoid transformation but are still separated by white lines that represent either remaining austenite with high carbon content or cementite. Judging by the number of these parallel lines, the number of plates was estimated to about 10 . It may be noted that all the white lines are parallel to each other but not to the overall shape of the packet, which is partly affected by internal eutectoid colonies or side layers. It seems that the habit plane of the primary plates of ferrite may have been different from the shape dominated by the eutectoid transformation.

Close inspection of Figure 13 reveals that the internal microstructure is changing on the left-hand side. A broad tip has developed, which covers the width of several plates in the interior. At its very top, the internal structure is too fine to be resolved and, further back, the white lines have already disappeared. Figures 14(a) and

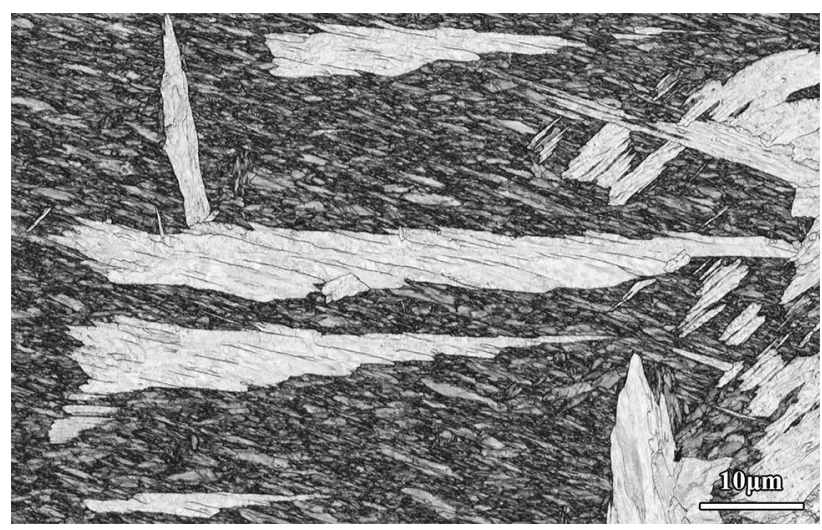

Fig. 9-Same region as Figs. 7 and 8 but with an EBSD quality map, which reveals some of the boundaries between the ferrite laths.

(b) show more developed examples from the same temperature. If this is a general phenomenon on the long edge of lathlike plates, then one should expect to observe it also in a section, which is almost in the main growth direction but not quite. When the section finally leaves a long packet on its side, it could pass through a layer formed on a long edge by widening. That kind of section is illustrated by case $\mathrm{c}$ in Figure 1 . This provides an explanation of how the top of the plate in Figure 14(c), which is a continuation of a longer packet in Figure 24(c) of Reference 7, can show the same change of microstructure as the cross sections in Figures 14(a) and (b).

The two bainite units in Figure 15(a) from $673 \mathrm{~K}$ $\left(400{ }^{\circ} \mathrm{C}\right)$ are packets of horizontal plates but cannot be seen because the interspaces have been transformed by the cooperative eutectoid reaction. However, lengths of the cementite lamellae give an indication of the initial widths of the interspaces. The packets have probably been sectioned between the directions of length and width of the primary plates, which would then have lengthened at an appreciable angle to the plane of polish. It is proposed that the finer lamellar structures at the two ends are the results of widening growth at the long edges, similar to what started at the vertical arrow in Figure 11. On the lower side of these two packets, they have contact to units of martensite, probably formed during the quench. The EBSD map in Figure 15(b) demonstrates that they belong to another bcc variant. The main parts of the units of bainite, including the fine lamellar structures, belong to a single bcc variant without much rotation. It appears that the fine ferrite plates have the same lattice orientation as the primary plates that cannot be distinguished. In Figure 11, there was a definite difference in orientation.

\section{DISCUSSION}

\section{A. Nucleation of Acicular Ferrite}

From the experience that there are combinations of sufficiently short time and high temperature where no bainite is observed in the inner parts of the austenite 

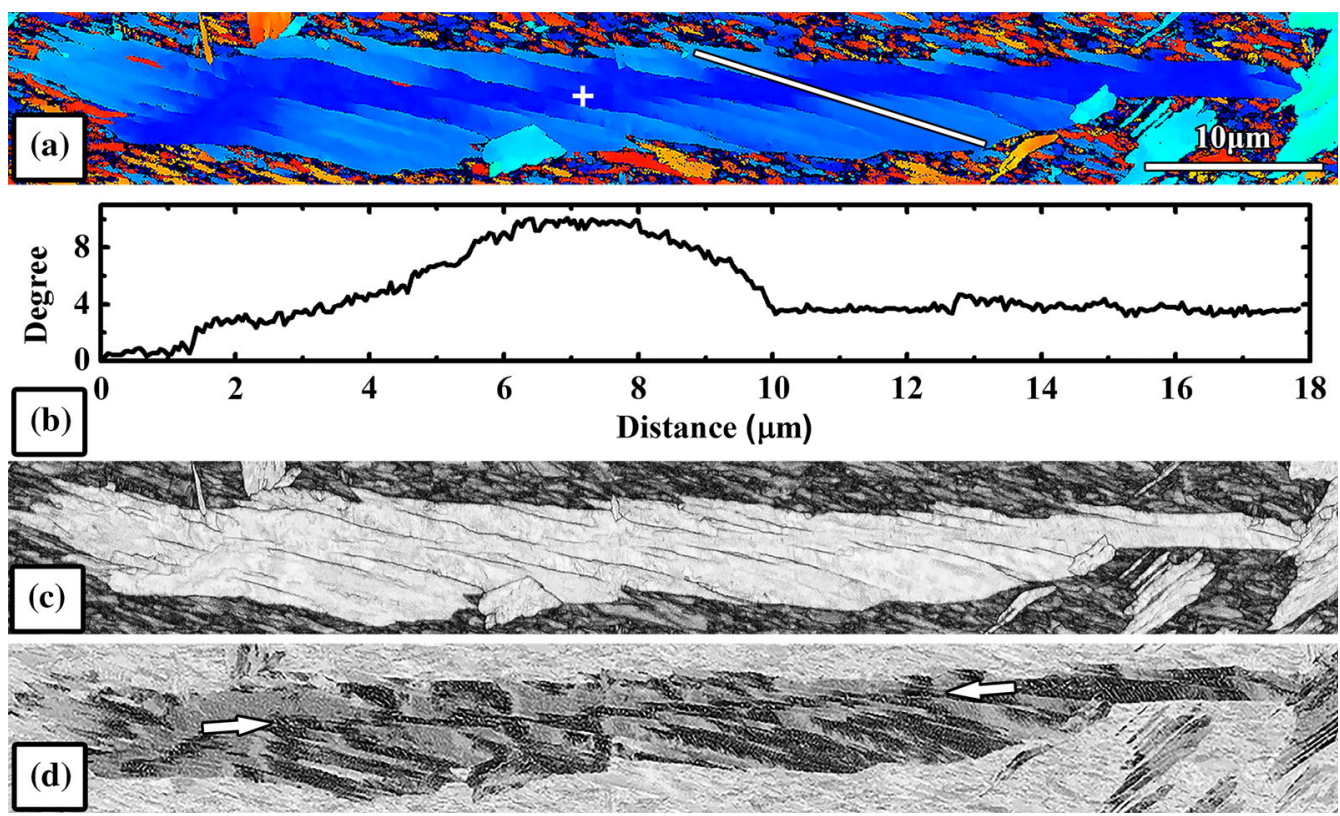

Fig. 10-High magnification for the largest packet in Fig. 8. (a) An EBSD misorientation map to a reference point, marked with a white cross. (b) Misorientation line scan analysis, with the white line marked in (a) from upper left to lower right, showing that widening growth is accompanied by some lattice rotation (maximum about $6 \mathrm{deg}$ ). (c) EBSD quality map and (d) ECCI contrast with two arrows indicating a stack of black, almost horizontal plates. They are proposed to have formed by lengthening growth.

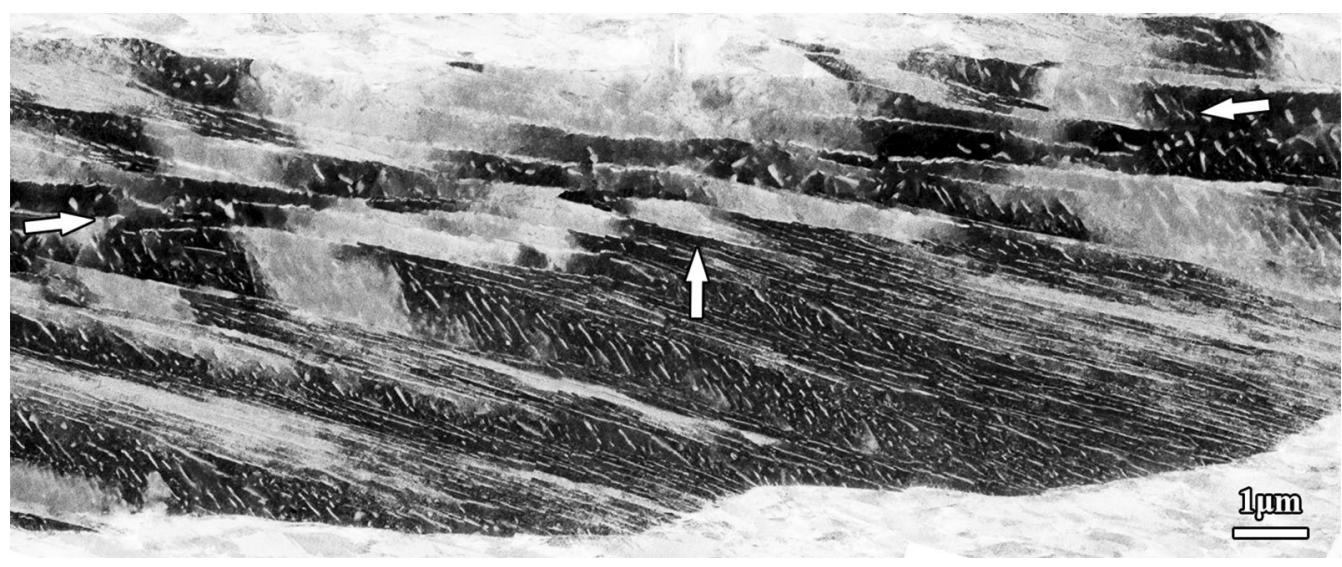

Fig. 11-Internal structure of the largest packet shown in Figs. 7 through 9, here under ECCI contrast. An imaginary line between the two horizontal arrows marks the parts formed before widening has started. These laths widened to both upper and lower sides with a change of growth direction. Widening on the lower side resulted in wider interspaces due to the change of direction, and one could see fine ferrite plates formed through branching from the original parts of ferrite laths, e.g., from the lath indicated by the vertical arrow. In other cases, the widened interspaces have transformed in a cooperative manner.

grains, it may be concluded that all bainite units have then nucleated on grain boundaries even if no contact is visible in the plane of polish. Groups of short, parallel plates are often observed and are identified as cross sections of packets of plates, and it can be safely concluded that at least the first plate to nucleate in a packet has done so in contact with a grain boundary. With reference to a micrograph, Aaronson and Wells ${ }^{[16]}$ proposed that the subsequent plates have been nucleated one by one in contact with previous ones and have obtained the same lattice orientation by sympathetic nucleation. This seems less likely because the plates in a group have not been reported, or observed in the present work, to be in contact with each other until the interspaces have been transformed, usually to a eutectoid mixture of ferrite and cementite. On the contrary, there are usually interspaces of various widths within a group. It is now proposed that the plates in a group have the same lattice orientation, because they have been nucleated on the same grain boundary and it gave preference to a particular orientation. ${ }^{[4]}$ It is, thus, implied that the plates in a packet have all nucleated on a grain boundary. It seems less likely that there would be another mechanism by which more plates with the same 

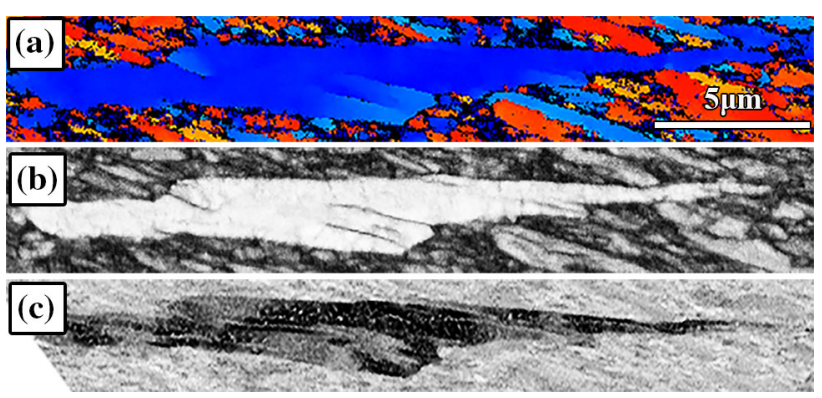

Fig. 12-High magnification for the lowest packet in Fig. 8, with (a) an EBSD misorientation map, $(b)$ an EBSD quality map, and $(c)$ ECCI contrast. Here, the major part of the volume of the entire packet formed through lengthening growth; widening growth has not yet developed much.

lattice orientation could also form on the side of a plate when it has grown away from the grain boundary. The observation that several fine plates are replacing a thicker one, e.g., as in Figures 11 and 14, is instead explained by branching.

A nucleation process for subunits, very similar to sympathetic nucleation, was proposed for explaining the moderate lengthening rates of bainitic ferrite with a hypothesized diffusionless growth mechanism. ${ }^{[17,18]}$ It was supported by reference to a very convincing micrograph. ${ }^{[19]}$ The thin end of the second longest packet in Figure 7 is very similar to that micrograph and also gives the impression that the last plate was nucleated on the side of the preceding one close to its end, and so on. This is illustrated well at the higher magnification in Figure 16 of the right-hand part of the next longest packet in Figure 7. However, it has already been explained that all plates in this packet, even starting from the group of short plates in a packet below the left-hand end of the packet, have formed in contact with a hidden grain boundary. It does not seem likely that there should be a different mechanism for the last few plates. Instead, it is proposed that the plates in a packet have formed one after another on a grain boundary, and it is difficult to avoid the conclusion that this is due to some influence from the preceding plate on the next one and that may be considered as a case of stimulated nucleation even though the nucleation occurs on the grain boundary, not on the side of a plate, as proposed for sympathetic nucleation. ${ }^{[6]}$

All the packets in Figures 7 through 9 seem to start from a straight line, and it was concluded that such a line actually exists on a nucleating grain boundary on a level away from the plane of polish. The most probable explanation is that it is a twin boundary because that would explain why there are not similar plates or packets on its other side, since the hidden grain boundary would have a different structure there although it would still belong to the grain shown on the plane of polish. It is an interesting question why the packets have thick ends close to the imaginary line, but this seems to indicate that the twin boundary actually stimulates the nucleation of a series of plates. For some reason, nucleation is then possible at some distance from the twin boundary and the plates become more and

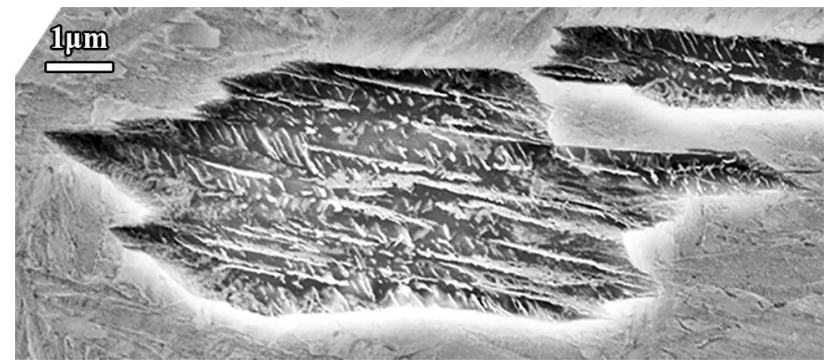

Fig. 13 - Cross section of a packet. Interspaces between the ferrite plates have mostly transformed through eutectoid reaction. The number of ferrite plates might be estimated by counting the number of long white thin lines, possibly cementite or remained austenite. SEM micrograph from an Fe-0.7C alloy held for $8 \mathrm{~s}$ at $723 \mathrm{~K}(450$ $\left.{ }^{\circ} \mathrm{C}\right)$.

more displaced sideways and the packets turn thinner. When looking for an explanation of this feature, it should be remembered that the most common shape of packets in cross sections is that the plates are stacked on top of each other, as in Figure 2. Normally, there does not need to be a twin boundary. Is it possible that the twin boundary has stimulated the formation of ferrite plates with an unusual orientation relation to the parent austenite, a relationship that favors sideways displacement when a prior plate nucleates the next one in the absence of a twin boundary? It is possible that a 3-D study could provide an answer, but this kind of packet is not very common and the chance of finding the hidden grain boundary is only 50 pet.

\section{B. Widening Growth}

Lengthening of ferrite laths occurs by constant movement of the short edge, presumably under diffusion of carbon over a constant effective diffusion distance. Thickening occurs under gradually decreasing advancement of the broad faces due to increasing diffusion distances of carbon. In contrast, it seems that one has not reached any understanding of widening, but, by definition, it would involve the movement of the two long edges.

In a preceding work ${ }^{[4]}$ on the morphology of proeutectoid ferrite in steels with 0.3 mass pct carbon, it was observed that widening of ferrite laths could be recognized by a change of growth direction. Protrusions were sometimes observed on the ends of plates in a cross section of a packet. In other cases, a small spike could develop from the end but in a new direction and, consequently, with a new habit plane. Such spikes could even grow to substantial lengths. These observations were interpreted as stages of the same process. It was as if the primary width had been stabilized at some stage and a second process was then starting up, which resulted in a ridge on the long edges of the lath.

Similar observations were made in the present work on the Fe-C alloy with 0.7 mass pct carbon, and in Figures 4(b) and (c), it was demonstrated that the ferrite lattice rotates when a ridge, with a new habit plane, starts to form. It was, thus, concluded that the central, dark blue band of plates in Figure 8 formed by 

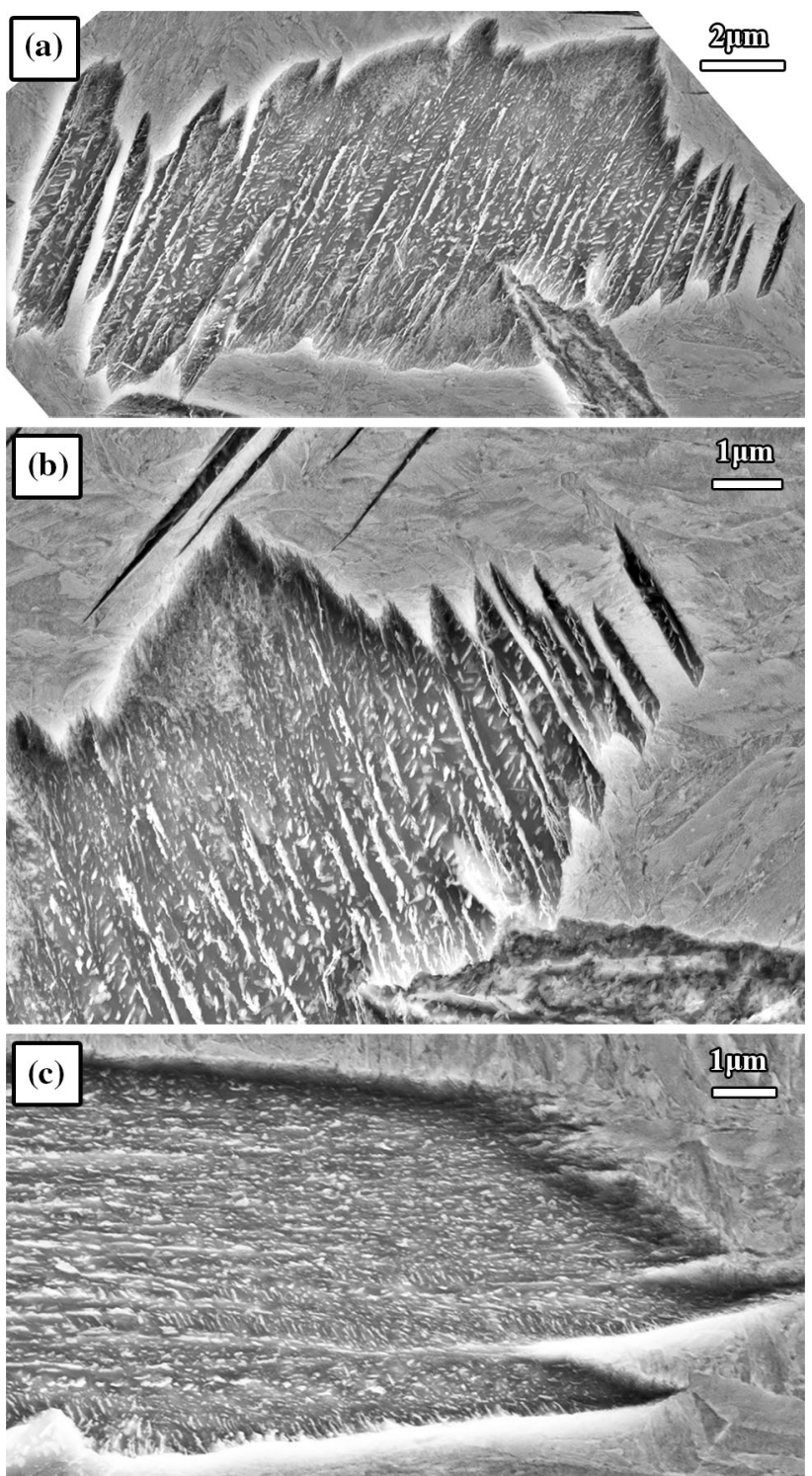

Fig. 14 - (a) A packet of lath-shaped ferrite plates sectioned close to their cross section. A spike has developed from the end of each lath and they have coalesced, resulting in a new ferrite/austenite interface as seen in both upper and lower sides of the packet. (b) Higher magnification of (a). (c) Another packet from the same specimen with a section close to the length dimension of the laths. SEM micrograph from an Fe-0.7C alloy after $8 \mathrm{~s}$ at $723 \mathrm{~K}\left(450{ }^{\circ} \mathrm{C}\right)$.

lengthening growth and retained the original width and lattice orientation. Rotation of the lattice then occurred on both sides but in different directions, and widening growth formed ferrite of the new orientation in the light blue areas. However, from Figure 8 or 10 , it was not immediately evident that the widening growth occurred in a new direction as it did in Figure 4. This apparent inconsistency was clarified by comparing Figures 10(a) and (d), and it was proposed that only the horizontal black parts in Figure 10(d) were formed by lengthening. Accepting that nucleation of acicular ferrite occurs on a grain boundary, it is proposed that the plate obtains its initial width by spreading along the grain boundary in connection to the nucleation event and, primarily, that
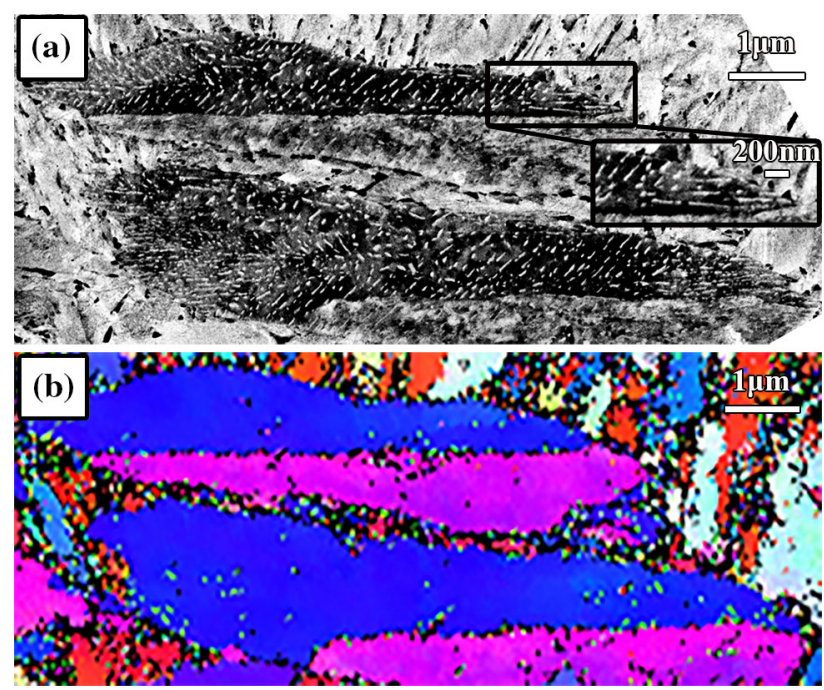

Fig. 15-Two packets of ferrite plates in an Fe-0.7C alloy held for $30 \mathrm{~s}$ at $673 \mathrm{~K}\left(400{ }^{\circ} \mathrm{C}\right)$. (a) ECCI micrograph. The number of plates is difficult to estimate due to the fine structure. One could see that the two ends of packets have grown through widening and formed groups of fine plates. (b) EBSD IPF map revealing that the martensite units belong to another variant of ferrite.

width is then transmitted to the lath when it forms by lengthwise growth.

The total width of the plates, which are best distinguished in Figure 10(c), is much larger than their central, dark blue band, seen as black in Figure 10(d) or 12 , and it is concluded that the other parts have formed by the movement of the long edges, i.e., by widening growth. It, thus, appears that the crystalline structure of the ferrite/austenite interface at the long edges was not very mobile, but, in some way, the structure was reorganized and could then move under transformation of the austenite lattice into ferrite. However, the growing ferrite in this new stage would obtain different crystalline orientation and habit plane.

It is an interesting question whether the widening already started at the base of the lath, i.e., in contact with the grain boundary. Once the protrusion forms there, it may spread along the long edge and form a rib. This is likely to be the case since observation of the protrusion in a 2-D section is not rare. Validation of this speculation might require 3-D observation down to the nucleating grain boundary and ideally with $3-D$ reconstruction of the widening. This may be a challenging task and is not the scope of this work.

Already from the lath shape, it is evident that lengthening is more rapid than widening even though the so-called point effect on diffusional growth could be the same. It may be concluded that the mobility of the edge around a lath varies from low for the long parts to high for the short parts. This situation has been approached by Aaronson et al. ${ }^{[20]}$ when they considered the role of transformation disconnections in ledgewise growth of plate-shaped transformation products and concluded that they could produce shape change. They emphasized that the disconnections in the ledges on the lateral sides, i.e., on the sides of the long edges, would 


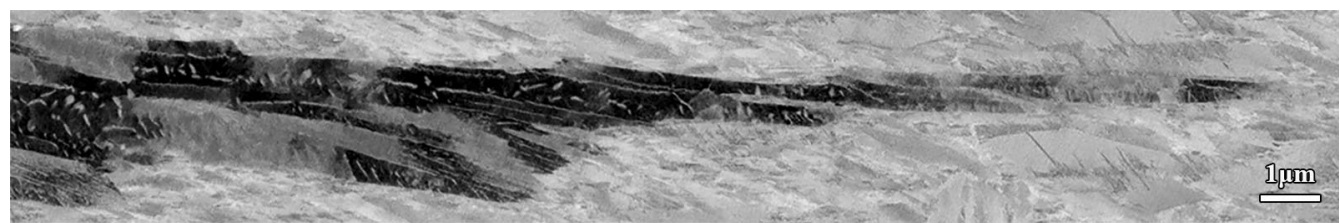

Fig. 16-Tip of the second longest packet shown in Figs. 7 through 9, which gives the impression that the packet is lengthening by forming new units at the top of the previous ones. It is proposed that such morphology is actually shown in its cross section, its true length dimension being roughly normal to the plane of polish.

automatically have a screw character. Hence, they postulated that lengthening should be faster than widening due to less strain incompatibility in that direction. They mainly discussed the thickening process, but their final figure depicts the lengthening and widening of a lath by the movement of a leading ledge, followed by a series of ledges. They did not discuss the fact that the leading ledge should create a layer of the new phase, which is surrounded by the parent phase on both sides. It should thus be an edge rather than a ledge. Furthermore, for the present case, where the habit plane and growth direction are different, the situation may be even more complex, but their approach seems promising.

\section{PROPOSALS}

Based on studies of iron alloys with 0.3 or 0.7 mass pct carbon, the following understanding has been reached for isothermal transformation to bainite in the upper temperature range.

1. The broad faces of laths mainly form by the advancement of the short edge. Its orientation and the direction of its movement characterize the habit plane.

2. The short edge of a lath develops early and in contact with a grain boundary.

3. The initial width of a lath is primarily determined by the length of the short edge as formed in contact with a grain boundary.

4. The structure of the long edges makes them less mobile, maybe not even spontaneously mobile.

5. At $773 \mathrm{~K}\left(500{ }^{\circ} \mathrm{C}\right)$, there may eventually be some movement of the long edges but with some rearrangement by which the structure of the edge is modified to give a different preferred growth direction and, hence, a different habit plane. This may develop into a ridge or even a thin plate attached to the long edge.

6. A similar ridge may form on the other long edge but in the opposite direction.

7. A considerable width can form by widening with a change of growth direction.

8. Packets of bainite plates are established in the nucleation process on a grain boundary.

9. Widening at $723 \mathrm{~K}\left(450^{\circ} \mathrm{C}\right)$ can result in finer mixtures of ferrite and austenite.

10. A thin packet of plates, with each one giving the impression of being nucleated close to the top of a prior plate, can originate by nucleation on a grain boundary.

11. It is proposed that crystallographic work should focus on the orientation dependence of the interfacial structure of the edges of laths.

\section{ACKNOWLEDGMENTS}

The authors acknowledge the financial support from VINNOVA, the Swedish Governmental Agency for Innovation Systems, the Swedish industry, and the KTH Royal Institute of Technology. The work was performed within the VINN Excellence Centre Her$\mathrm{o}-\mathrm{m}$. The $\mathrm{Fe}-\mathrm{C}$ alloy investigated in this work was kindly offered by Dr. I. Zuazo, Arcelor Mittal Maizieres Research (S.A.). Professor G. Purdy is acknowledged for an important discussion. The authors are grateful for the help from Associate Professor P. Hedström during the work of EBSD characterization. J. Yin thanks the China Scholarship Council (CSC) for sponsorship of his study.

\section{OPEN ACCESS}

This article is distributed under the terms of the Creative Commons Attribution 4.0 International License (http://creativecommons.org/licenses/by/4.0/), which permits unrestricted use, distribution, and reproduction in any medium, provided you give appropriate credit to the original author(s) and the source, provide a link to the Creative Commons license, and indicate if changes were made.

\section{REFERENCES}

1. F.B. Pickering: Transformations and Hardenability in Steels, Climax Molybdenum Co., Ann Arbor, MI, 1967, pp. 109-29.

2. Y. Ohmori, H. Ohtani, and T. Kunitake: Trans. ISIJ, 1971, vol. 11, pp. 250-59.

3. H.K.D.H. Bhadeshia: Bainite in Steels, 3rd ed., Maney Publishing, WakeField, United Kingdom, 2015.

4. J. Yin, M. Hillert, and A. Borgenstam: Metall. Mater. Trans. A, 2017, vol. 48A, pp. 1425-43.

5. J. Yin, M. Hillert, and A. Borgenstam: Metall. Mater. Trans. A, 2017, vol. 48A, pp. 1444-58.

6. H.I. Aaronson: The Decomposition of Austenite by Diffusional Processes, V.F. Zackay and H.I. Aaronson, eds., Interscience, New York, NY, 1962, pp. 387-546. 
7. J. Yin, M. Hillert, and A. Borgenstam: Metall. Mater. Trans. A, 2017, vol. 48A, pp. 4006-24.

8. R.E. Hackenberg, D.P. Nordstrom, and G.J. Shiflet: Scripta Mater., 2002, vol. 47, pp. 357-61.

9. K.M. Wu and M. Enomoto: Scripta Mater., 2002, vol. 46, pp. 569-74.

10. M.V. Kral and G. Spanos: Metall. Mater. Trans. A, 2005, vol. 36A, pp. 1199-1207.

11. L. Cheng, Z.G. Li, and K.M. Wu: ISIJ Int., 2008, vol. 48, pp. $830-34$

12. L. Cheng, X.L. Wan, and K.M. Wu: Mater. Charact., 2010, vol. 61, pp. 192-97.

13. L. Cheng and K.M. Wu: Acta Mater., 2009, vol. 57, pp. 3754-62.

14. K.M. Wu, Y. Inagawa, and M. Enomoto: Mater. Charact., 2004, vol. 52, pp. 121-27.
15. H.E. Exner: in Metallography and Microstructures, vol. 9, ASM Handbook, G.F. Vaner Voort, ed., ASM INTERNATIONAL, Materials Park, OH, 2004.

16. H.I. Aaronson and C. Wells: Trans. AIME, 1956, vol. 206, pp. 1216-23.

17. J.M. Oblak and R.F. Hehemann: Transformations and Hardenability in Steels, Climax Molybdenum Co., Ann Arbor, MI, 1967, pp. 15-38.

18. H.K.D.H. Bhadeshia and D.V. Edmonds: Metall. Trans. A, 1979, vol. 10A, pp. 895-907

19. H.K.D.H. Bhadeshia and D.V. Edmonds: Acta Metall., 1980, vol. 28 , pp. $1265-73$.

20. H.I. Aaronson, T. Furuhara, M.G. Hall, J.P. Hirth, J.F. Nie, G.R. Purdy, and W.T. Reynolds, Jr: Acta Mater., 2006, vol. 54, pp. 1227-32. 